\title{
Trastornos menores de salud en una muestra de estudiantes de la Universidad de Barcelona
}

\author{
Minor health disorders in a sample of students of the University \\ of Barcelona
}

Icart Isern $\mathrm{MT}^{1}$, Pulpón Segura $\mathrm{AM}^{2}$, Icart Isern $\mathrm{MC}^{3}$

\begin{abstract}
Departamento de Enfermería de Salud Pública, Salud Mental y Materno-Infantil. Escuela de Enfermería. Universidad de Barcelona
\end{abstract}

Introducción: conocer la prevalencia de algunos trastornos menores de salud (TMS) en una muestra de estudiantes de la Universidad de Barcelona (2004-05). Material y métodos:. El estudio se realizó en las facultades de Ciencias Económicas, Farmacia, Medicina, Psicología y Químicas y Escuela de Enfermería de la Universidad de Barcelona. Se empleó un diseño observacional, descriptivo y transversal mediante un cuestionario mixto administrada a 600 estudiantes durante el mes de diciembre de 2004. Las variables estudiadas corresponden a los siguientes TMS: insomnio, estreñimiento, cefalea, dolor osteomuscular, tics nerviosos, onicofagia y tricotilomania.

Resultados:. El sexo femenino representa el $64 \%$ de la muestra; la media de edad de los encuestados es de 22,7 (DE: 3,27; IC 95\%: 22,43-22,96). Las mujeres acumulan el 67,79\% de los 1.245 TMS identificados. Por sexo, se observan diferencias estadísticamente significativas en las prevalencias de cefalea, dolor osteomuscular y tricotilomania que afectan en mayor medida al sexo femenino. La onicofagia (264; 21,20\%; IC 95\%: 18-24,4) es el más extendido de los TMS, seguido de la cefalea (233; 18,72\%; IC 95\%: 15,6-21,84). los estudiantes de Psicología presentan el mayor
Correspondencia:

M. Teresa Icart Isern

Feixa Llarga s/n. 08907 - L'Hospitalet de Llobregat

Tel: 93402 4241. Fax: 934024297

E-mail: mticart@ub.edu número, seguidos de los de Ciencias Económicas; los que presentan un menor número son los de Farmacia y Químicas.

Conclusiones: El sexo femenino presenta la mayor prevalencia de TMS. La onicofagia y la cefalea son los principales TMS identificados en la muestra. Los estudiantes de Psicología acumulan el mayor número de TMS, siendo los que presentan más cefalea, dolor osteomuscular y tricotilomania.

Palabras clave:: Estudiantes universitarios. Insomnio.

Estreñimiento. Cefalea. Dolor osteomuscular. Tics nerviosos. Onicofagia. Tricotilomania.

Background: To determine the prevalence of certain minor health disorders (MHD) in a sample of students from the University of Barcelona (2004-05).

Methods: The study was carried out in the faculties of Economic Sciences, Pharmacy, Medicine, Psychology and Chemistry, and in the School of Nursing, all of the University of Barcelona. An observational, descriptive and cross-sectional design was used and involved the administration of a mixed questionnaire to $\mathbf{6 0 0}$ students during December 2004. The studied variables correspond to the following MHD: insomnia, c onstipation, headache, bone and muscle pain, nervous tics, onychophagia and trichotillomania.

Results: $64 \%$ of the sample was female and the mean age of interviewees was 22.7 (SD: 3.27; Cl 95\%: 22.43-22.96). Women accounted for $67.79 \%$ of the 1,245 MHD identified. There were significant sex differences in the prevalence of bone and muscle pain 
and trichotillomania, which mostly affected females. Onychophagia (264; $21.20 \%$; Cl 95\%: 18-24.4) was the most common MHD, followed by headache (233;

18.72\%; Cl 95\%: 15.6-21.84). Psychology students reported the highest number of MHD, followed by those from Economic Sciences; the smallest percentages were presented by students from Pharmacy and Chemistry.

Conclusions: MHD are most prevalent among women. Onychophagia and headache were the main MHD identified in the sample. Psychology students report the largest number of MHD, the most common being migraine, bone and muscle pain and trichotillomania.

Key words: university students, insomnia, constipation, headache, bone and muscle pain, nervous tics, Onychophagia, trichotillomania.

\section{INTRODUCCIÓN}

Los estilos de vida y los hábitos tóxicos en la población universitaria han sido objeto de diversos estu$\operatorname{dios}^{1-4}$ en los que se identifica la prevalencia de algunos factores asociados al consumo de sustancias tóxicas como el tabaco, el alcohol y las drogas de síntesis. En otros trabajos se analiza, de forma específica, la relación entre los estilos de vida y la salud mental ${ }^{5,6}$. Sin embargo, sólo destacamos un estudio relacionado con la prevalencia de trastornos menores de la salud (TMS) en la población universitaria que fue realizado, en 1987, en la Universidad Autónoma de Barcelona y en el que se comparaba la prevalencia de algunos de estos trastornos (insomnio, estreñimiento, dolor de cabeza, amenorrea, etc.) entre los estudiantes de psicología respecto a la población general ${ }^{7}$.

El interés por el estudio de estos TMS se debe, al menos en parte, a su amplia prevalencia y a la incomodidad que generan en el normal desarrollo de las actividades cotidianas.

En este sentido, el insomnio que es el trastorno más común del sueño, afecta entre el 20 y $40 \%$ de la población española y un $17 \%$ sufre de insomnio grave 8 . Aunque se presenta de las distintas formas (dificultad para conciliar el sueño, despertarse con frecuencia por la noche o muy temprano) se habla de insomnio cuando las noches en vela se prolongan más allá de tres semanas ${ }^{8}$.
El estreñimiento que se define como la emisión retardada e infrecuente (menos de tres deposiciones a la semana) de heces anormalmente duras, secas y a menudo difíciles de expulsar, alcanza una prevalencia del $2,5 \%$ en varones y del $5 \%$ en mujeres españolas ${ }^{9}$.

La cefalea constituyen uno de los síntomas más frecuentes en la práctica clínica con una prevalencia del $20 \%$ en mujeres y del $7 \%$ en varones. En la mayoría de casos se trata de cefalea tensional ocasionada por la contracción prolongada de los músculos del cuello, hombros o cabeza, asociada a posturas incorrectas, al estrés o al cansancio ${ }^{10,11}$.

En cuanto al dolor osteomuscular, tiene origen multifactorial, su fisiología no está clara y agrupa varias entidades (dolor miofacial, síndromes músculo-tendinosos, fibromialgia, etc. ${ }^{12}$. Según un estudio realizado en Cataluña ${ }^{13}$ la prevalencia del dolor en la población general, en los 6 meses anteriores al estudio, fue del 78,6\%.

Otros TMS que a primera vista revisten menos importancia que los anteriores son: los tics nerviosos, la onicofagia y la tricotilomania; no obstante, pueden generar incomodidad y cierto malestar entre quienes los padecen.

Los tics nerviosos afectan a un $20 \%$ de niños pequeños (3 veces más en niños que en niñas) y suelen desaparecen pasada la adolescencia. Pueden ser motores (parpadear, hacer gestos faciales, chuparse un dedo, mover las manos, etc.) o vocales (carraspeo, quejidos, olfateos, etc. ${ }^{14}$. La onicofagia o hábito compulsivo de comerse la uñas es más frecuente en el sexo femenino ${ }^{15}$.

Finalmente la tricotilomania o tricología está considerada como un trastorno del control de los impulsos que consiste en arrancarse el pelo de forma recurrente; siendo la cabeza la parte más afectada pero puede abarcar distintas partes del cuerpo (cejas, pestañas, axilas o pubis), al igual que la onicofagia, se sitúa entre los 3 y 7 años. Al tratarse de trastornos evolutivos tienden a desaparecer con la edad, por lo que no suelen observarse en el adulto quien, por otra parte, suele minimizar la importancia del problema ${ }^{16}$.

El estudio de estos TMS entre los estudiantes universitarios interesa porque algunos pueden provocar disminución del rendimiento académico (insomnio, cefalea), también pueden traducir situaciones de ansiedad o estrés emocional (tics nerviosos, onicofagia, tricotilomanía) y, obviamente, pueden ser síntomas de enfermedades de diversa gravedad (dolor osteomuscular, cefalea, estreñimiento).

El objetivo de nuestro estudio es conocer la prevalencia de algunos trastornos menores de salud 
en una muestra de estudiantes de la Universidad de Barcelona; también, se estudia la relación de estos trastornos con el sexo y la carrera que cursan.

\section{MATERIAL Y MÉTODOS}

Ámbito. El presente estudio tuvo lugar entre los meses de diciembre de 2004 y febrero de 2005 en las Facultades de Ciencias Económicas, Farmacia, Medicina (Campus de Bellvitge), Psicología, Química y en la Escuela de Enfermería de la Universidad de Barcelona.

Diseño. Se utilizó un diseño observacional, descriptivo y transversal.

Sujetos. El estudio se realizó con una muestra no probabilística, de conveniencia, integrada por 600 alumnos, 100 de cada facultad, siendo el único criterio de inclusión el estar matriculado en la respectiva carrera.

Instrumento. Para la obtención de los datos se empleó un cuestionario que fue administrado por estudiantes de enfermería, previamente entrenadas, que se desplazaron a las facultades objeto de estudio. El cuestionario estaba compuesto por tres preguntas abiertas y siete cerradas de respuesta dicotómica, siendo el tiempo destinado para responderlo de aproximadamente 4 minutos. El modelo final fue precedido por una prueba piloto que permitió mejorar la formulación de algunas preguntas.

La variable principal del estudio engloba algunos trastornos menores de salud que pueden afectar a los estudiantes universitarios. Dichos trastornos incluyen: insomnio, estreñimiento, cefalea, dolor osteomuscular, tics nerviosos, onicofagia y tricotilomania.

Figura 1. Distribución del sexo según carrera $(n=600)$

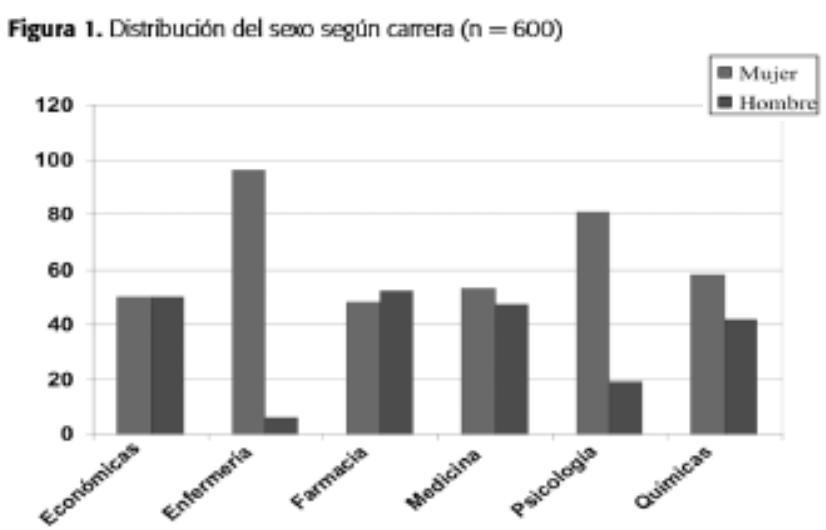

Análisis. Para el análisis de las variables se aplicaron estadísticos descriptivos y pruebas de «2 para el contraste de hipótesis, asumiendo un error de primera especie del $5 \%(p<0,05)$; también se calcularon los intervalos de confianza del 95\%. La aplicación informática utilizada fue el programa estadístico SPSS v. 11.5.

\section{RESULTADOS}

En la muestra, las mujeres encuestadas fueron $384(64 \%)$ y los varones 216 (36\%) observándose diferencias estadísticamente significativas $(« 2=78,03$; $\mathrm{gl}=5 ; \mathrm{p}=0,001)$ a favor del sexo femenino. La distribución del sexo según carrera (fig.1) muestra que el sexo femenino predomina en todas las carreras estudiadas excepto en farmacia.

La edad media de la muestra fue de 22,7 años (DE: 3,27; IC 95\%: 22,43-22,96) y en su distribución por sexo, se apreciaron diferencias estadísticamente significativas $(\mathrm{t}=2,095 ; \mathrm{gl}=598 ; \mathrm{p}=0,037)$. La media de edad más elevada corresponde a los estudiantes de Farmacia (tabla 1).

Tabla 1. Distribución de la edad según sexo y carrera de la muestra estudiada $(n=600)$

\begin{tabular}{|c|c|c|c|c|c|}
\hline & $x$ & DS I & $\begin{array}{r}\text { Edad } \\
\text { Mediana }\end{array}$ & Rango & IC $95 \%$ \\
\hline \multicolumn{6}{|l|}{ Sexo } \\
\hline Mujer $(n=384)$ & 22,49 & 3,3 & 22 & $18-45$ & $22,16-22,82$ \\
\hline Hombre $(n=216)$ & 23,07 & 3,2 & 23 & $18-38$ & $22,64-23,50$ \\
\hline \multicolumn{6}{|l|}{ Carrera } \\
\hline Económicas & 22,12 & 3,2 & 21,5 & $18-33$ & $21,48-22,76$ \\
\hline Enfermería & 22,48 & 4,21 & 21 & $18-45$ & $22,48-23,32$ \\
\hline Farmacia & 23,56 & 2,82 & 23 & $20-32$ & $23-24,12$ \\
\hline Medicina & 22,98 & 2,12 & 23 & $18-27$ & $22,56-23,40$ \\
\hline Psicología & 22,68 & 3,9 & 22 & $18-38$ & $21,91-23,45$ \\
\hline Químicas & 22,36 & 2,83 & 22 & $18-38$ & $21,80-22,92$ \\
\hline
\end{tabular}

Tabla 2. Distribución de los trastornos de salud en la muestra estudiada

\begin{tabular}{l|r|r|r|r}
\hline \multirow{2}{*}{ Trastornos menores } & \multicolumn{3}{|c|}{ Casos } & \multicolumn{3}{c}{ IC 95\% } \\
& \multicolumn{2}{|c|}{$\mathrm{N}$} & $(\%)$ & \\
\hline Insomnio & 175 & 14,05 & $11,27-$ & 16,83 \\
Estreñimiento & 168 & 13,49 & $10,76-$ & 16,22 \\
Cefalea & 233 & 18,72 & $15,60-$ & 21,84 \\
Dolor osteomuscular & 222 & 17,83 & $14,77-$ & 20,89 \\
Tic nervioso & 79 & 6,36 & $4,41-$ & 8,31 \\
Onicofagia & 264 & 21,20 & $18-$ & 24,40 \\
Tricotilomania & 104 & 8,35 & $18-$ & 10,56 \\
Otros & 1.245 & 100 & & \\
\hline
\end{tabular}


Tabla 3. Distribución de los trastornos menores según sexo

\begin{tabular}{l|r|r|r|r|r}
\hline Trastornos menores & \multicolumn{4}{|r|}{ Mujer $(\mathrm{n}=384)$} & \multicolumn{2}{|r|}{ Hombre $(\mathrm{n}=216)$} & \\
& $\mathrm{n}$ & $(\%)^{*}$ & $\mathrm{n}$ & $(\%)^{*}$ & $\mathrm{P}$ \\
\hline Insomnio & 114 & 65,1 & 61 & 34,9 & $\mathrm{NS}$ \\
Estreñimiento & 115 & 68,5 & 53 & 31,5 & $\mathrm{NS}$ \\
Cefalea & 163 & 70 & 70 & 30 & 0,01 \\
Dolor osteomuscular & 156 & 70,3 & 66 & 29,7 & 0,009 \\
Tic nervioso & 47 & 59,5 & 32 & 40,5 & $\mathrm{NS}$ \\
Onlcofagia & 166 & 62,9 & 98 & 37,1 & $\mathrm{NS}$ \\
Tricotilomania & 83 & 79,8 & 21 & 20,2 & 0,001 \\
\hline Total & 844 & 401 & & & \\
\hline
\end{tabular}

* Porcentaje en relación al sexo

El total de TMS registrados asciende a 1.245 , siendo la onicofagia, seguida de la cefalea los trastornos más extendidos (tabla 2).

Por sexo, las mujeres acumulan el 67,79\% (IC 95\%: 64,06-71,52) y los hombres el $32,21 \%$ (IC 95\%: 28,48-35,94) de los trastornos estudiados. Al considerar la distribución de cada TMS con relación al sexo, se verificaron diferencias estadísticamente significativas en la presencia de cefalea $(\ll 2=5,86 ; \mathrm{gl}=1 ; \mathrm{p}=$ $0,015)$, dolor osteomuscular $(\ll 2=6,04 ; \mathrm{gl}=1$; $\mathrm{p}=0,009$ y tricotilomania $(\ll 2=13,644 ; \mathrm{gl}=1$; $\mathrm{p}=0,001)$ a expensas de una mayor frecuencia en el sexo femenino (tabla 3 ).

Por carrera, Psicología acumula el mayor número de trastornos menores, seguida de Ciencias Económicas (tabla 4). Los estudiantes de Económicas presentan la mayor prevalencia de insomnio $(52 ; 29,7 \%)$ y tics nerviosos $(42 ; 53,2 \%)$; los de Medicina, la de estreñimiento $(46 ; 27,4 \%)$ y onicofagia $(56 ; 21,2 \%)$; a los de Psicología, corresponden los mayores porcentajes de cefalea, dolor osteomuscular y tricotilomania. Las carreras de
Enfermería, Farmacia y Químicas no sobresalen en la presencia de ninguno de los TMS estudiados.

\section{DISCUSIÓN}

El predominio del sexo femenino entre la población universitaria, se confirma en nuestro estudio; no obstante, el resultado podría haber sido diferentes de haber incluido otras carreras (Ingeniería, Matemáticas, etc.).

La media de edad, superior en la carrera de Farmacia, puede deberse al azar ya que se trata de muestras no probabilísticas de tipo accidental o de conveniencia. Esta es una limitación del estudio a considerar en la valoración de los resultados obtenidos y que impide cualquier generalización. Otra limitación que conviene tener en cuenta, deriva de los propios conceptos sobre los que se preguntaba y que se pueden entender de modo diferente según las creencias y los conocimientos inherentes a cada carrera.

En cuanto a los TMS, y al igual que en el estudio de Bayes y Riba 7 la onicofagia y la cefalea ocupan el primer y segundo lugares respectivamente, mientras que la prevalencia del insomnio, los tics nerviosos y la tricotilomania es ligeramente superior en nuestro estudio ya citado.

Si comparamos la prevalencia del insomnio, observamos que en la población española 8 es más del doble respecto a nuestro estudio; también es superior en la estadounidense ${ }^{16}$.

Cabe destacar que la mayoría de adultos se encuentra bien con 6 o 7 horas de sueño, con las que se recupera el organismo, se protege el sistema inmune y se mantienen las funciones cerebrales ${ }^{8}$. Para los estudiantes, la consecuencia más importante del insomnio es la baja concentración con la consecuente falta de atención, sobre todo por la mañana.

Tabla 4. Distribución de los trastornos menores según carrera

\begin{tabular}{|c|c|c|c|c|c|c|c|c|c|c|c|c|c|c|c|c|}
\hline \multirow[t]{2}{*}{ Carrera } & \multicolumn{2}{|c|}{ Insomnio } & \multicolumn{2}{|c|}{ Estreñimiento } & \multicolumn{2}{|c|}{ Cefalea } & \multicolumn{2}{|c|}{$\begin{array}{c}\text { Dolor } \\
\text { osteo-muscular }\end{array}$} & \multicolumn{2}{|c|}{ Tic nervioso } & \multicolumn{2}{|c|}{ Onicofagia } & \multicolumn{2}{|c|}{ Tricotilomania } & \multicolumn{2}{|c|}{ Total } \\
\hline & $\mathrm{n}$ & (\%) & $\mathrm{n}$ & (\%) & $\mathrm{n}$ & (\%) & & $(\%)$ & $\mathrm{n}$ & (\%) & $\mathrm{n}$ & (\%) & $\mathrm{n}$ & $(\%)$ & & $(\%)$ \\
\hline Económicas & 52 & 29,7 & 36 & 21,4 & 40 & 17,2 & 19 & 8,6 & 42 & 53,2 & 39 & 14,8 & 9 & 8,7 & 237 & 19,04 \\
\hline Enfermería & 19 & 10,9 & 19 & 11,3 & 39 & 16,7 & 44 & 19,8 & 3 & 3,8 & 50 & 18,9 & 19 & 18,3 & 193 & 15,50 \\
\hline Farmacia & 16 & 9,1 & 25 & 14,9 & 27 & 11,6 & 19 & 8,6 & 3 & 3,8 & 35 & 13,3 & 13 & 12,5 & 138 & 11,09 \\
\hline Medicina & 21 & 12 & 46 & 27,4 & 27 & 11,6 & 58 & 26,1 & 3 & 3,8 & 56 & 21,2 & 3 & 2,8 & 214 & 17,18 \\
\hline Psicología & 30 & 17,1 & 24 & 14,3 & 63 & 27 & 76 & 34,2 & 22 & 27,8 & 42 & 15,9 & 47 & 42,2 & 304 & 24,41 \\
\hline Químicas & 37 & 21,2 & 18 & 10,7 & 37 & 15,9 & 6 & 2,7 & 6 & 7,6 & 42 & 15,9 & 13 & 12,5 & 159 & 12,78 \\
\hline Total & 175 & 100 & 168 & 100 & 233 & 100 & 222 & 100 & 79 & 100 & 264 & 100 & 104 & 100 & 1245 & 100 \\
\hline
\end{tabular}


Los porcentajes del estreñimiento en nuestra muestra son similares a los hallados por Bayes ${ }^{7}$ pero claramente superiores a la población general. Esta diferencia podría explicarse por la valoración subjetiva del problema, que ignora la definición médica del síntoma 9 y emplea la más popular de que "lo normal es una deposición diaria"; en ese sentido, los estudiantes de Medicina son los que presentan una mayor prevalencia de estreñimiento. En realidad se sabe poco acerca de los factores de riesgo del estreñimiento en la población joven ${ }^{17}$ ya que es un problema más estudiado en ancianos; llama la atención que en el estudio de Bayes y en el nuestro se obtuvieran cifras que casi triplican a las de la población general.

De modo similar a otros estudios ${ }^{10,11}$, en los que la cefalea triplica su presencia en el sexo femenino, también en el nuestro duplica a la registrada por el sexo masculino. En la mayoría de casos su mecanismo es poco conocido (cefaleas primarias), pero en otros se asocia a procesos patológicos conocidos (cefaleas secundarias) por lo que, en caso de persistencia, se recomienda su estudio.

El dolor osteomuscular es otro TMS cuya valoración, por tratarse de una sensación, es compleja y subjetiva. De forma general, el dolor está influido por factores sociales, culturales, experiencias previas, grado de atención, ansiedad, etc. No obstante, las cifras obtenidas en nuestro estudio son netamente inferiores a las del estudio de Bassols y cols en la población catalana ${ }^{18}$ aspecto que podría atribuirse a que la mayoría de estudiantes son jóvenes y sanos. Llama la atención que el dolor osteomuscular y la cefalea tengan mayor prevalencia en la misma carrera (Psicología).

En cuanto a los tics nerviosos, la tricotilomania y la onicofagia observamos cifras más o menos similares a las obtenidas por Bayes ${ }^{7}$. Se trata de TMS que afectan a cualquier etapa de la vida ${ }^{14}$, y aunque sus causas no se han identificado, se suelen asociar a cierto grado de estrés o conflicto emocional.

En conclusión, la onicofagia seguida de la cefalea son los TMS con mayor prevalencia entre los estudiantes. El sexo femenino presenta más del doble del TMS respecto al sexo masculino. Los estudiantes de Psicología presentan el mayor número de TMS y duplican a los de Químicas con el menor porcentaje entre las carreras estudiadas.

\section{AGRADECIMIENTOS}

A los alumnos de la Escuela de Enfermería: Albuxech L, Álvarez A, Asencio P, Gil M, Paredes N, Pérez S, Zafra V, que administraron los cuestionarios.

Este estudio fue parcialmente financiado por el Departamento de Enfermería de Salud Pública, Salud Mental y Materno-Infantil. Escuela de Enfermería. Universidad de Barcelona

\section{BIBLIOGRAFÍA}

1. Heras T, García A, Hernández C, Ballestín MC, Nebot M. Tabaquismo en los estudiantes de enfermería de Catalunya. Hábitos, actitudes y conocimientos. Gac Sanit. 1997;11(6):267273

2. Cobas E, Lista MJ, Álvarez M, Prenas S, González C. Estilos de vida de los estudiantes de enfermería: una oportunidad para la acción. Metas Enferm. 2003; VI (3): 61-64

3. Iturrioz U, Erice A, Zupira MJ, Barandiarán X, Cascante MT. Hábitos insanos en estudiantes de enfermería. Enferm Científ. 2002 sep-oct; 246-247:48-57

4. Frojan MX, Rubio R. Salud y hábitos de vida en los estudiantes de la UAM. Clínica y Salud. 1997; 8(2):357-381

5. Aguinaga E, Guillen E, Notivol MP, Pérez D, Aguinaga I. Salud mental y estilo de vida de los estudiantes de enfermería de la Universidad de Navarra. Enferm Clínica. 1996;6(3):108-112

6. Fajardo B, Cruz A. Ansiedad. Situaciones y estímulos ansiógenos en estudiantes de Enfermería. Rev ROL Enferm. 1992;XV(162):31-38

7. Bayés R, Riba MD. Trastorns lleugers de salud en una població universitària. Gac Sanit. 1987;1(2):63-67

8. Anónimo. Insomnio [Acceso 13 de julio, 2005]. Disponible en: http://www.el-mundo.es/la revista/num135/textos/in1.htm

9. Pizarro M, Rancaño I. Estreñimiento. Guías Clínicas 2001. [Acceso 20 de junio, 2005]. Disponible en http://www. fisterra.com/guias2/estreñimiento.asp

10.Centro Neurológico Dr. García Trujillo. ¿Qué es la cefalea? [Acceso 20 de junio, 2005]. Disponible en: http//:www.silinfo.com/neuro

11.Ezpeleta D. Cefalea e Internet. Neurología. 2002;17 (supl 2): 1-13

12.Rodríguez-Hernández JL. Osteomuscular and rheumatic pain. Rev Soc Esp Dolor. 2004;11(2):56-64

13.López de Castro R, Rodríguez FJ. Méndez I, Mancebo R, Gómez R. ¿Existen diferencias en la percepción del dolor entre varones y mujeres? Aten Primaria. 2003;31(1):18-22

14.Pereira J. Tics nerviosos. [Acceso 20 de junio, 2005]. Disponible en: http//jpereira.eresmas.net/tics.html

15.Azeli YA. Onicofagia. [Acceso 20 de junio, 2005]. Disponible en: http//:podium.es/podium/anom8.htm 
16.Anónimo. Dificultad para dormir. [Acceso 17 de julio, 2005]. Disponible en: http//: www.nlm.nih.gov/medlineplus/spanish/ ency/article/003210.htm

17. Bixquet M, Fillat O, López C, Sastre A, Serrano P. Factores demográficos y dietéticos en el paciente con estreñimiento cró- nico. Revista de la Sociedad Valenciana de Patología Digestiva. 2002; 21(2):82-86

18. Bassols A, Bosch F, Campillo M, Cañellas M, Baños JE. An epidemiological comparison of pain complains in the general population of Catalonia (Spain). Pain. 1999; 83:9-16 